\title{
Zum Kircheneinigungsversuch des Jahres 1439.
}

Solange wir für die geschichtliche Erkenntnis der religiösen und kirchlichen Verhältnisse des durch den Fall Konstantinopels im Jahre 1453 vernichteten und damit als lebendiges Glied aus der Reihe der europäischen Staaten endgültig ausgeschiedenen oströmischen Reiches auf die Mignesche Patrologie mit ihren meist so mangelhaft hergerichteten und in ihrer Vollständigkeit oft durch den blofsen Zufall bestimmten Texten angewiesen sind; solange vor allem eine Ausgabe der sämtlichen Schriften der beiden Hauptwortführer der damaligen griechischen Kirche, Markos Eugenikos und Gennadios Scholarios, insbesondere ihrer Briefe, nichts als ein frommer Wunsch ist: so lange wird es nötig sein, auf den schriftstellerischen Nachlafs der beiden grofsen Hellenen zurückzukommen, ihn nach Möglichkeit zu ordnen, Dunkelheiten in demselben aufzuhellen und unzureichend Bekanntes oder unbekannt Gebliebenes hervorzuziehen, zu erläutern und in den ihm gebührenden geschichtlichen Zusammenhang einzuordnen.

Frühere Darlegungen ergänzend und weiterführend, kehre ich zu dem Kircheneinigungsversuche des Jahres 1439 zurück. Mehrere der unmittelbar hierbei oder in weiterer Folge in Betracht kommenden Umstände bedurfen eingehenderer Darstellung. Bekannt ist, wio dio beiden Führer und Berater des hellenischen Volkes Bessarion und Markos Eugenikos in ihren Ansichten und Überzeugungen sich schroff gegenüberstanden. Bessarion hielt an der Meinung fest, dafs das Volk, wenn es sich nicht der päpstlichen Herrschaft unterwürfe und die rechtgläubige Lehre nach dem Willen des Papstes änderte, mit der Beugung unter das türkische Joch dem Islam verfallen würde. Markos dagegen zog das türkische Joch dem Papsttum vor, das zugleich die freiwillige Verwerfung des väterlichen Glaubens in sich schlofs. Jener glaubte, dafs, wenn die Kirche des Morgenlandes der römischen sich untergeordnet habe, das Abendland zur Befreiung des griechischen Volkes von der türkischen Herrschaft herbeieilen würde; dieser verwarf solche Hoffnung entweder als eine völlig eingebildete und nichtige, oder als für das 
Volk schädliche und zu tödlichem Verderben führende, wenn anders das Abendland je sie verwirklichen sollte. Deshalb ermahnte Bessarion die in Florenz zum Zwecke der Kirchenvereinigung erschienenen griechischen Geistlichen, die vom Papst herrührende Einigungsurkunde der Synode zu unterschreiben. Er mahnte um so dringlicher, als er versicherte, dafs infolge der Versagung der Unterschriften sämtliche Strafen des unentrinnbar heraufziehenden entscheidenden Völkerkampfes auf der Widerstrebenden Häupter fallen würden, - er selbst sei unschuldig daran. Der Tag, an welchem diese schweren Worte Bessarions in Florenz vor Kaiser Johannes VIII Paläologos, seinen Hofbeamten und den orthodoxen Geistlichen geredet wurden, war der 4. Juli 1439. An demselben Tage liefs der Papst den Griechen die verhängnisvolle Urkunde zur Unterschrift vorlegen. Die Lage war furchtbar, die Zeit zu Verhandlungen unwiderbringlich verstrichen, ein Aufschub nicht möglich. Jetzt mufste notwendig das eine von beiden, entweder das Joch des Sultans vorgezogen und damit, nach Bessarions Annahme, der christliche Hellenismus geopfert, oder des Papstes Joch, und damit, nach Markos' Meinung, der rechtgläubige Hellenismus aufgegeben werden. Ein drittes gab es nicht. In der Versammlung, die sich in ihrer Gesamtheit Bessarions Mahnworten gegenüber stumm verhielt, safs auch Markos, der Exarchos, schweigend in tiefem Schmerze, wie der Augenzeuge Syropulos erzählt. Mit zitternder Hand unterschrieben alle, vom Kaiser anfangend, das vorgelegte Schriftstück und verleugneten damit feierlich den Glauben ihrer Väter. Verstohlen, wie ihrer That sich schämend, blickten die, welche unterschrieben hatten, auf Markos. Dieser, in entscheidender Stunde von unbeugsamem Mut und auch den Mächtigsten dieser Welt gegenüber von heldenhafter Unerschrockenheit, stand auf und verliefs festen Schrittes die Versammlung mit den berühmten Worten: Ich unterschreibe nicht, komme, was da wolle. Wie grols der Ärger des Papstes Eugenios IV war, als er erfuhr, Markos von Ephesus habe die Einigungsurkunde nicht unterschrieben, geht aus der von Syropulos überlieferten Äufserung desselben hervor: Nichts haben wir ausgerichtet ( der Lateiner gegen den Wortführer der Griechen war so grofs, dafs Kaiser Johannes sich genötigt sah, bei seiner Abreise von Florenz nach Venedig im September 1439 Markos von Ephesus in seine besondere Obhut zu nehmen, um ihn vor Hinterhalten und Nachstellungen der Lateiner zu schützen; in seinem eigenen Schiffe liefs er den ephesinischen Erzbischof von Venedig mit nach Konstantinopel zurückkehren.

Drei Monate nach der am 1. Februar 1440 erfolgten Rückkehr von der Kirchenversammlung liefs es der Kaiser sich angelegen sein, 
dem in Florenz gestorbenen Patriarchen Joseph einen Nachfolger zu bestellen. Bei der so überaus feindseligen Stimmung gegen Rom war es kein Wunder, dafs Markos von Ephesus in erster Linie in Vorschlag kam. Da dieser aber unbedingt ablehnte, so entschied sich der Kaiser für den Bischof Metrophanes von Kyzikos, einen entschlossenen Freund Roms und der Kirchenvereinigung. Das geschah am Himmelfahrtsfest, den 4. Mai des Jahres 1440. Als zehn Tage darauf der neue Patriarch seines Amtes waltete, versagten ihm die Bischöfe Dorotheos von Trapezunt, Markos von Ephesus, Antonios von Heraklea ihre Mitwirkung und zugleich auch die Kirchengemeinschaft. Der Kaiser, sehr ungehalten hierüber, suchte sie zwar zur kirchlichen Mitwirkung zu zwingen, aber noch an demselben Tage, am Pfingstfest, flohen Markos und Antonios, um der thatsächlichen Anerkennung der ihnen verhafsten Vereinigung zu entgehen, heimlich aus der Stadt, Antonios, nach der Überfahrt auf dem Landwege ununterbrochen Tag und Nacht weiter eilend, zu seiner Bischofsstadt, dem pontischen Heraklea, Markos über Prusa, bis wohin wohl beide Flüchtlinge sich zusammenhielten, nach Ephesus.

Unmittelbar in die Tage zwischen Himmelfahrt und Pfingsten 1440 versetzt uns der von Demetrakopulos in seinem Verzeichnisse der

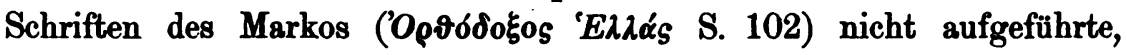
aber a. a. O. S. 106 aus einer Münchener Handschrift (256, S. 155) mitgeteilte Brief des Markos an den auf der Insel Imbros lebenden Hieromonachos Theophanes. Dieser hatte eine an den Kaiser gerichtete Schrift wider die Florentiner Kirchenversammlung verfalst, sowie eine Abhandlung über den Ausgang des hl. Geistes und das ungesäuerte

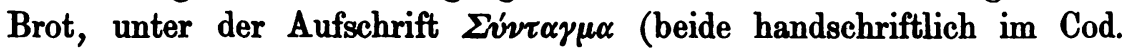
Monac. 256). Beide Schriften sandte er an den noch in Konstantinopel befindlichen Markos von Ephesus mit der Bitte, wenn er sie gut heifse, sie persoulich dem Kaiser zu ubergeben. Markos, mit Inhalt und Absicht der eingesandten Schriften durchaus einverstanden, trug mit Rücksicht auf die Zeitumstände Bedenken, sie dem Kaiser zu überreichen. $\mathbf{E r}$ schrieb vielmehr an Theophanes jenen Brief, der für die Kenntnis der Zeitumstände sehr wichtig ist. Diese sind in demselben so deutlich wie möglich gekennzeichnet. Man ist von Italien zurückgekehrt, Markos wohl und munter. Durch Theophanes' Sendung ist ihm in diesen Zeiten der Trübsal und des Abfalls eine wahre Herzstärkung zuteil geworden. Der neue Patriarch ist bereits erwählt, ein Mietling in des

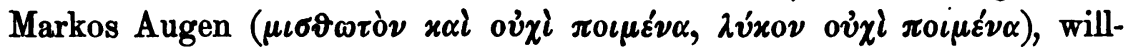
fährig und wohlgeschickt, um, wie man hofft, den verderblichen lateinischen Glauben aller Seelen einzupflanzen. Noch sind keine feindseligen Maßsregeln wider die Rechtgläubigen ergriffen, Markos fürchtet 


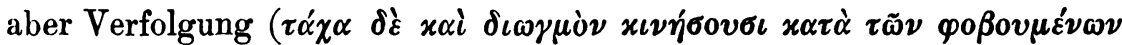

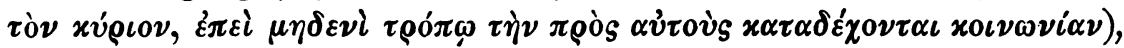
die Gegensätze haben sich schon so weit zugespitzt. Die Zeiten des Redens und der wissenschaftlichen Verhandlung sind vorüber, man drängt zur That, und die Rechtgläubigen müssen sich auf Leiden und Kampf gefafst machen. In einem so sturmdrohenden, unheilschwangeren Zeitpunkt würde die Uberreichung der wackeren Schriften des Theophanes vom Kaiser, der übrigens durch Markos' Hinweis auf Jesaja 3, 4

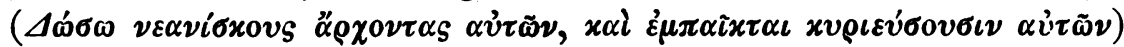
schlimm gekennzeichnet ist, nichts als Spott und Hohn eintragen. Darum unterlärst es Markos. Die Abfassungszeit dieses Briefes kann nur in jenen Tagen unmittelbar vor dem Pfingstfest des Jahres 1440 gesucht werden. In dieselbe Zeit der Entscheidung, als Metrophanes den Patriarchenstuhl bestiegen und Markos, seiner Überzeugung folgend, Konstantinopel verlassen hat und nunmehr die Leiden, von denen er geredet, auch über ihn hereingebrochen sind, führt uns ein anderer, gleichfalls von Demetrakopulos (a. a. O. S. 102) aus derselben Münchener Handschrift Nr. 256 mitgeteilter Brief ein, der, nach seinem Eingang und seinem Ton zu schliefsen, höchst wahrscheinlich an denselben Theophanes gerichtet, seiner Unterschrift zufolge nur wenige Wochen später als der erste, nämlich am 16. Juni 1440, geschrieben ist. Der kaiserliche Unwille hat danach den der Kircheneinigung so hartnäckig sich widersetzenden Ephesier wohl zu finden gewufst. Markos erzählt, wie er notgedrungen die Hauptstadt verlassen und sich nach Ephesus ( $\left.\varepsilon i_{S} \tau \eta \dot{\nu} \varepsilon x x \lambda \lambda \eta \sigma i \alpha \nu \mu o v\right)$ begeben habe. Aber auch dort hat er nicht die gewünschte Ruhe gefunden. Krankheit und Anfeindungen von Gegnern haben ihn wieder aus der Stadt getrieben. Er scheint zunächst nicht den Seeweg eingeschlagen zu haben, um in die Stille irgend eines der Athosklöster zu fliehen, sondern die Gemeinschaft von Gesinnungsgenossen im Lande nördlich von Ephesus, vielleicht gar Antonios in Heraklea aufgesucht zu haben. Denn er erwähnt in seinem Briefe, er sei, in der Absicht zum Athos zu gelangen, über Kallipolis nach Lemnos gekommen. Kallipolis liegt nun aber auf dem thrakischen Chersones am Hellespont; Markos scheint also die Propontis, vielleicht von Nikomedia oder Kyzikos aus, durchschifft zu haben, während die Fahrt, von Ephesus unternommen, Lemnos vielleicht, Kallipolis aber niemals berühren konnte. In Lemnos war es, wo den streitbaren Bischof der kaiserliche Haftbefehl erreichte; er ward festgenommen und in die Verbannung geschickt. Wohin? deutet er in seinem Schreiben mit keinem Worte an. Von dem Orte seiner Verbannung aber scheint der Brief an Theophanes jedenfalls geschrieben. 
Hier hat er auch erfahren, dafs der Bischof von Monembasia, Dositheos, dessen Namen er freilich nicht nennt, von den am Ruder befindlichen Freunden der Lateiner zum Bischof von Athen gewählt ist, und dafs dieser nun sich beeilt, lateinisch gesinnte Genossen in die kirchlichen Ämter zu bringen. Warnend vor der Gemeinschaft mit solchen Leuten, erhebt Markos seine Stimme am Schlufs des Briefes, der mit seinen wohlgelungenen Anklängen an Paulus' Galaterbrief (besonders 5, 2. 3) bei Theophanes, dem treuen Schüler des Isidoros ( $\tau \tilde{\eta} s \dot{\alpha} \lambda \eta \vartheta \varepsilon i \alpha s$ qi $\lambda_{0 S}$

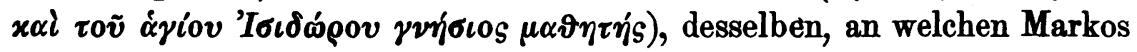
die von Boissonade zuerst 1844 (Anecd. Nov. Paris. S. 349-362) veröffentlichte Schrift $\Pi_{\varepsilon \rho i}$ ő $\omega \nu ~ \zeta \omega \tilde{\eta} s$ richtete, des Eindrucks nicht verfehlt haben wird.

Markos hat nur drei Jahre diese stürmischen Zeiten überlebt. Er starb, wie ich mehrfach nachgewiesen ${ }^{1}$ ), im Jahre 1443. An diesem Ergebnis meiner Untersuchung vermag mich auch eine Mitteilung Nikephoros Kalogeras' nicht irre zu machen. Ich hatte demselben meinen

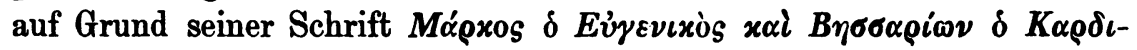
vád cs (vgl. Byz. Z. IV 145-153) verfafsten, in der „Neuen kirchlichen Zeitschrift" V 1002-1020 erschienenen Aufsatz "Markos Eugenikos und Kardinal Bessarion" zugesandt, worauf er mir aus Athen den

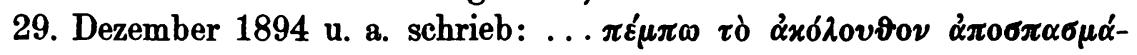

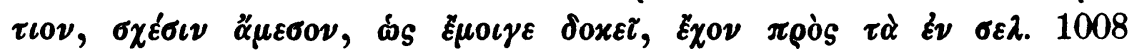

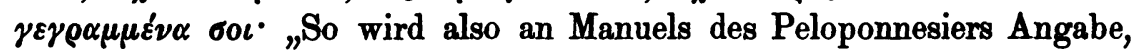
d. h. an dem Jahre 1443 als Todesjahr des Markos festgehalten werden

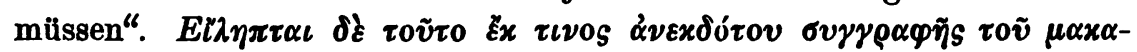

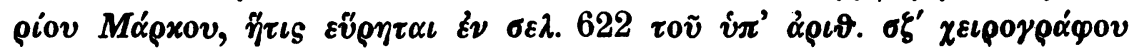

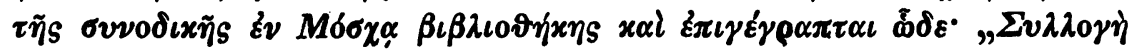

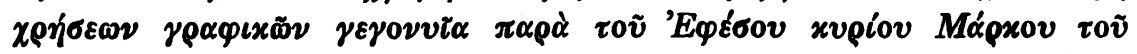

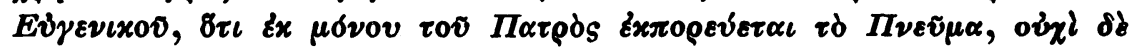

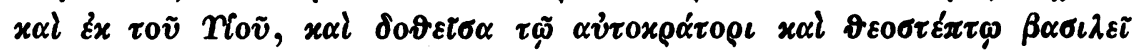

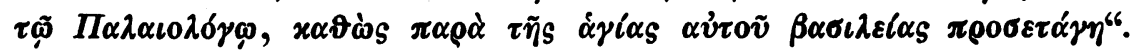

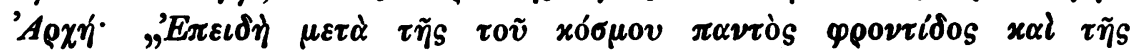

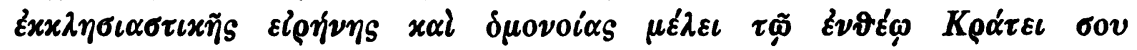

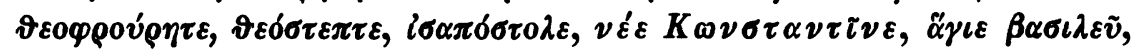

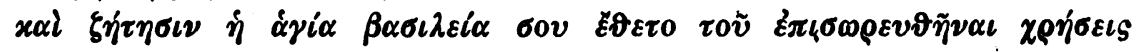

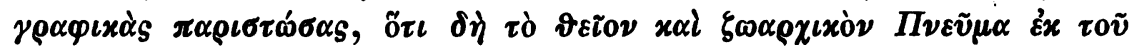

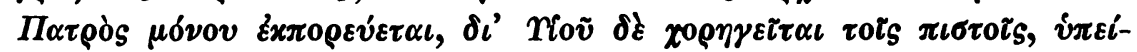

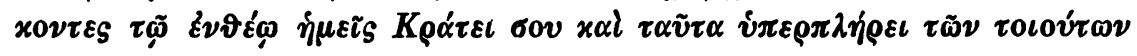

1) Zeitschr. für Kirchengesch. XII 92-97. Neue kirchl. Zeitschr. V 10061009. Byz. Zeitschr. IV 150-153. 


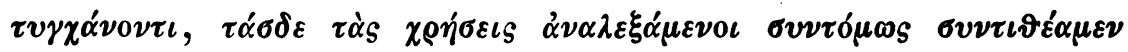
$\varkappa \tau \lambda .{ }^{6}$ Mit dem von Kalogeras selbst gesperrt gegebenen Ausdruck

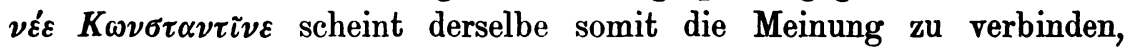
Markos habe die Regierung Konstantinos' XI, d. h. das Jahr 1448, noch erlebt. Er würde damit der Ansicht L. Hellers (Herzogs Realencyklopädie IX, 1858, S. 44) - und ebenso Gafs' (Realencyklopädie, 2. Aufl. 1881, S. 292) - zuneigen, der, wohl einfach auf Fabricius (Bibl. Gr. ed. Harl. XI, S. 670) gestützt, Markos im Jahre 1447 ge-

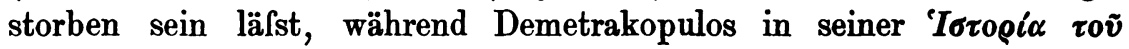

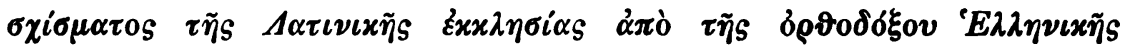
(Leipzig 1867), S. 147/148, Anm. 1 die wiederholt von mir betonten

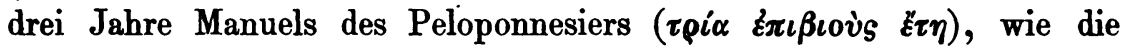
von ihm dazugesetzte Klammer ( heblich überschreiten zu dürfen geglaubt hat, aber in seinem Werk

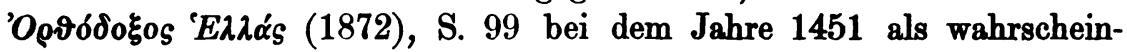
lichem Todesjahre des Markos stehen geblieben ist. Dafs eine solche Ausdehnung der Lebenszeit des ephesinischen Bischofs unzulässig ist und anderen geschichtlichen Nachrichten widerspricht, glaube ich bewiesen zu haben. Jenes überschwengliche Beiwort in der Anrede vย́

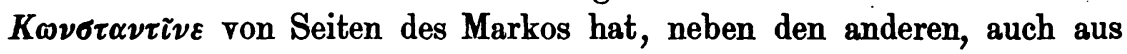
den Anreden des Nikolaos von Methone an Kaiser Manuel Komnenos uns ganz geläufigen, wie überhaupt die ganze Sammlung von Beweisstellen für die griechische Lehre, dafs der hl. Geist vom Vater, nicht aber auch vom Sohne ausgeht, deren Überschrift uns Kalogeras aus dem Cod. Mosqu. $\sigma \xi^{\prime}$ mitteilt, nur dann einen Sinn, wenn wir die Schrift im Auftrage Kaiser Johannes' VIII Paläologos (1425-1448) verfafst denken. Sie kann eigentlich nur zu einer Zeit entstanden sein, als man sich in Byzanz zur Konzilsfahrt nach Italien rüstete und abermalige theologische Vorbereitung für die in Aussicht stehenden Erörterungen mit den abendländischen Theologen vonnöten schien. Da mochte der Kaiser den überspannten Erwartungen der Hellenen als ein neuer Konstantinos ${ }^{1}$ ) erscheinen, der, wie der alte Imperator einst zu

1) Auch wenn wir Markos nicht mit besonderen geschichtlichen Kenntnissen ausgerüstet denken, so stand doch Kaiser Konstantinos als der erhabene Gründer der Stadt und hochverdiente Schirmherr der Kirche in Byzanz in besonderem Ansehen. Schaute doch auf der Mitte des grofsen, nach ihm benannten Marktes sein ehernes Standbild von jener gewaltigen Porphyrsäule auf das Volk von Konstantinopel hernieder, die so, wie sie der am 5. April 1101 in sie gefahrene Blitzstrahl äufserlich zugerichtet, noch heute als „verbrannte Säule“ dasteht, die letzte Zeugin längst entschwundener Pracht. Vgl. die Abbildung der Säule in Hertzbergs „Geschichte der Byzantiner und des Osmanischen Reiches", Berlin 
Nikäa (325), jetzt dort in Italien alle Zwietracht zu bannen und die Kircheneinheit in griechischem Sinne herbeizuführen gewillt und imstande war. Unter Konstantinos XI (1448-1453) lagen die Verhältnisse schon wesentlich anders, und es ist schwer einzusehen, welchen Zweck damals noch jene Sammlung gehabt haben sollte. Doch bleiben wir bei dem Jahre 1439, dem Jahre des Einigungsversuchs, noch einen Augenblick stehen.

Wie Georgios Scholarios aus einem anfänglichen Begünstiger der Kirchenvereinigung unter Markos Eugenikos' Einflufs zu einem überzeugten Gegner derselben wurde ${ }^{1}$ ), das habe ich in meinem Aufsatz „Zu Georgios Scholarios" (Byz. Z. IV 561-580) eingehend dargelegt. Wie aber stand es mit Kaiser Johannes' innerster Überzeugung in diesem Punkte? Wenn wir hören, dafs er bei seinem Aufenthalt in Italien die meiste Zeit mit nichtigen Vergnügungen, besonders mit Jagdausflügen hinbrachte, so werden wir an einen tieferen, seine Überzeugung und sein Handeln bestimmenden Ernst kaum zu glauben geneigt sein. Wir wissen, dafs er die Konzilsfrage gegen den ausdrücklichen Rat. seines weisen Vaters Manuel in Angriff nahm. Und wie hat er sich diesen väterlichen Warnungen gegenüber verhalten? Berüchtigt sind die lũgnerischen Worte, die der Unglückliche in Florenz vor den griechischen Theologen und den Männern des kaiserlichen Hofes redete, die alle gleichermalsen starr vor Staunen standen und auf die päpstliche Urkunde sahen, die zur Unterschrift vorlag: „Dies Werk der Einigung", sprach er nach Syropulos; Geschichte des Florentiner Konzils IX 60, „hat nicht mit mir seinen Anfang genommen, mein

1883, S. 8 mit der Anmerkung auf S. 19. Dafs an den ehernen Schirmherrn auf der Säule sich damals schon abergläubige Vorstellungen knüpften, geht aus Johannes Dukas (Kap. 39) hervor. Ein Schwärmer oder Betrüger, heifst es, hătte geweissagt, dafs die Türken eines Tages in die Stadt dringen und die Romer bis zur Konstantinssăule verfolgen würden; dies aber sollte das Ende aller ihrer Nöte und Drangsale sein. Ein Engel mit einem Schwerte in der Hand würde vom Himmel herabschweben und das Reich samt der himmlischen Waffe einem armen, am Fufse der Säule sitzenden Manne mit der Aufforderung übergeben, das Volk des Herrn zu rächen. Augenblicklich würden bei diesen belebenden Worten die Türken fliehen und die siegreichen Römer sie aus ganz Anatolien und bis an die Grenzen von Persien treiben.

1) Durch nichts sind wir befugt, mit Gibbon (History of the decline and fall of the Roman empire, London 1848, Band VIII Kap. 68, S. 288 Anm. h), der nur Renaudot. (vgl. Byz. Z. IV 563, Anm, 1) zu folgen scheint, Gennadios der „Doppelzüngigkeit seines Charakters" (the duplicity of his character) zu zeihen. Gibbon war s. Z. in diesen Fragen noch ganz aufserordentlich unzureichend unterrichtet. Erst jetzt vermögen wir in ihnen klarer zu sehen und jeden der handelnden Männer zu seinem Rechte kommen zu lassen. 
kaiserlicher Vater begann schon mit seiner Vorbereitung ... er trachtete danach, die Einigung zu vollenden, als eine gute;... es war ihm aber nicht beschieden, sie vollendet $\mathrm{zu}$ sehen. Deswegen trug er mir es auch auf, sie zu vollenden. Sein Werk ist es, und als in seinem Auftrage verrichte ich selbst dies hier. Und nicht nur er trachtete danach, sondern auch alle Einsichtigen und die Lehrer unserer Kirche, unter denen ich auch den Herrn Joseph (Bryennios) getroffen habe, der behauptete, jenes Mittel der Einigung und zwar als ein unfafsbares zu besitzen." Gerade die entgegengesetzte Ansicht betreffs des Konzils hegte sein Vater sein Leben lang, und es war eine dreiste Lüge, dafs er den erbitterten Gegner Roms, Joseph Bryennios, als einen Gesinnungsgenossen derer bezeichnete, welche die Zusammenberufung eines griechisch-römischen Konzils betrieben. Sollen wir diese Winkelzüge für wohlüberlegte Äufserungen byzantinischer Staatsweisheit halten und aus diesem Grunde etwa milder darüber urteilen? Gleichviel, eins steht fest: trotz aller Gewaltmafsregeln, von denen oben die Rede war, ist Kaiser Johannes sehr bald von Reue über den verhängnisvollen Schritt, den er gethan, erfafst worden. Schon in dem oben erwähnten Briefe an Theophanes vom 16. Juni 1440 bezeugt Markos Eugenikos ${ }^{1}$ ):

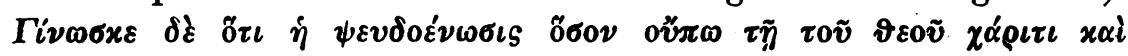

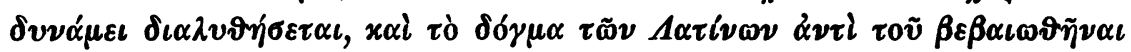

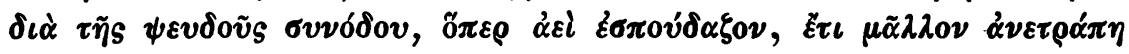

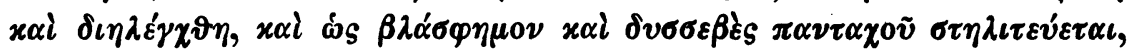

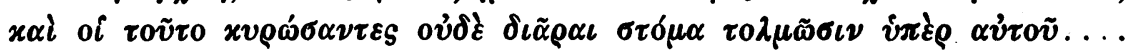

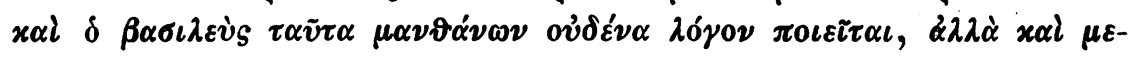

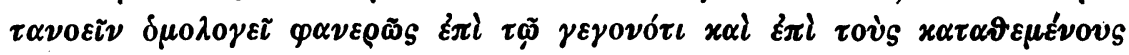

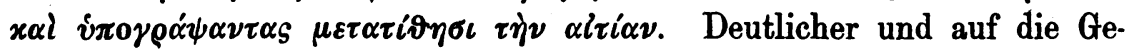
danken des Kaisers tiefer eingehend sind die von der Reue desselben zeugenden, zeitlich jedenfalls späteren Worte des Georgios Scholarios, welche Dositheos von Jerusalem in seiner Geschichte der Jerusalemischen Patriarchen ${ }^{2}$ ) aus einer Schrift desselben gegen den Zusatz der

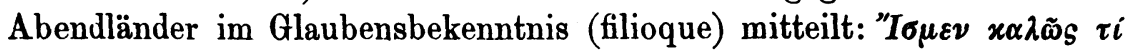

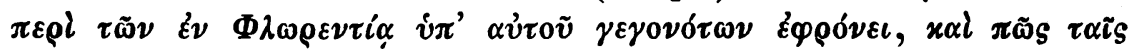

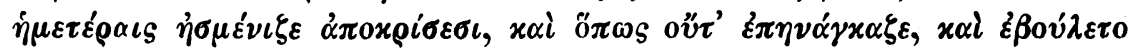

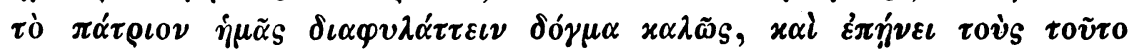

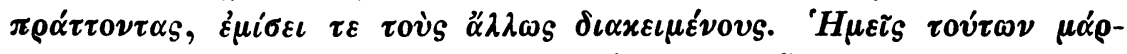

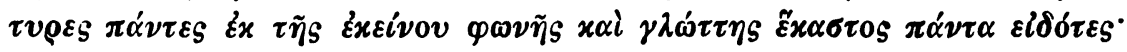

1) Cod. Mosq. 256 S. 279, vgl. Zeitschrift für Kirchengeschichte XII S. 106.

2) Buch X Kap. 13 nach Demetrakopulos' „Geschichte der Kirchenspaltung“ S. 160. 


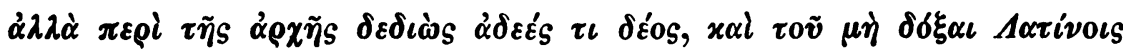

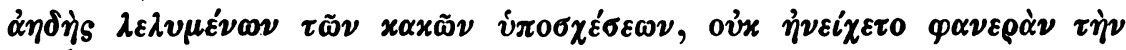

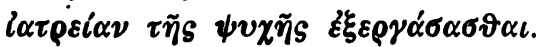

Blicken wir jetzt auf die weiteren Folgen des Einigungsversuchs, insbesondere die Stellung, die Georgios Scholarios demselben gegenüber eingenommen hat. Noch genauere Einzelheiten, als ich schon in dem oben angeführten Aufsatz gegeben, werden dem künftigen Geschichtbeiber dieser Zeiten nicht unwillkommen sein.

Ich sagte, die Zeiten Konstantinos' XI seien hinsichtlich der grofsen Kirchenfragen schon wesentlich andere gewesen als die seines Bruders Johannes VIII. Der Einigungsversuch des Jahres 1439, dessen wurde man sich ziemlich allgemein bewufst, war schon bei Lebzeiten des letzteren im wesentlichen gescheitert. Kaiser Johannes hatte vor seinem Tode auf die volksverhafste Mafsregel einer Vereinigung mit den Lateinern verzichtet, was sollte nun werden? Konstantinos berief im Jahre 1450 eine Synode, welche sich in der Sophienkirche versammelte. Anwesend waren die Patriarchen Philotheos von Alexandria, Ananias von Antiochia, Theophanes von Jerusalem und zahlreiche Geistliche sowie hervorragende Laien. Die erste Handlung, welche die Synode vornahm, war, dafs man den Patriarchen der Hauptstadt, Gregorios, der seit 1445 im Amte war, wegen seiner den Lateinern so entgegenkommenden Haltung zur Rechenschaft zog. Gregorios entfloh nach Rom, wo er später auch gestorben ist. Sodann verwarf man die Florentiner Kirchenvereinigung als eine trügerische und erzwungene sowie im Widerspruch mit den ökumenischen Synoden stehende und erklärte die dort gegebenen Unterschriften, weil gleichfalls erzwungen, für nichtig und unverbindlich. Kaiser Konstantinos geriet nun aber durch die drohende Haltung der Türken in immer grölsere Not, und diese Not war es, welche ihm den letzten Versuch der Schmeichelei und Verstellung auferlegte. Eiligst sandte er Andronikos Bryennios an Papst Nikolaus V, um Hilfe gegen die Türken zu erbitten; denn die Hilfe, die man durch Abschlufs der Florentiner Einigung zu erhalten gehofft hatte, war bisher ausgeblieben. Der kaiserliche Gesandte war angewiesen, in das Begehren zeitlicher Hilfe die Zusicherung geistlichen Gehorsams einfliefsen zu lassen; seine Vernachlässigung der Kirche wurde mit den dringenden Sorgen des Staates entschuldigt. Der Papst, der den Gedanken an eine wirkliche Kirchenvereinigung noch nicht aufgegeben hatte, schickte sofort den Kardinal Isidoros, den früheren Erzbischof von Rufsland, ab, der im Herbst des Jahres 1452 in Konstantinopel eintraf, freilich ohne die geringste Hilfe oder eine Aussicht auf solche. „Die abendländischen Fürsten waren in endlose, 
heimische Kämpfe verwickelt, und der römische Papst zürnte wegen der Falschheit und Hartnäckigkeit der Griechen. Statt zu ihren Gunsten die Waffen und Schätze Italiens zu verwenden, hatte Nikolaus $V$ ihren nahen Untergang geweissagt, und seine Ehre hing von der Erfüllung der Prophezeiung ab. Vielleicht besänftigte ihn der äufserste Grad ihrer Not, aber sein Mitleid kam zu spät, seine Bestrebungen waren schwach und unwirksam, und Konstantinopel gefallen, bevor die Geschwader von Genua und Venedig aus ihren Häfen segeln konnten. Selbst die Fürsten von Morea und der griechischen Inseln zeigten kalte Parteilosigkeit; die genuesische Kolonie zu Galata unterhandelte über einen Sondervertrag, und der Sultan liefs sie in der trügerischen Hoffnung, dafs seine Milde sie den Sturz des Reiches überleben lassen würde. Eine Schar Plebejer und einige byzantinische Grofse entzogen sich niedriger Weise der Gefahr ihres Vaterlandes, und der Geiz der Reichen verweigerte dem Kaiser und sparte für die Türken die geheimen Schätze, womit sie zu ihrer Verteidigung ganze Heere von .Söldnern hätten anwerben können. Der dürftige und alleinstehende Kaiser rüstete sich indessen, seinem furchtbaren Feinde zu begegnen; aber wenn sein Mut der Gefahr gleichkam, war seine Stärke doch dem Kampfe nicht gewachsen." 1 )

Gennadios Scholarios befand sich damals als Mönch - nicht in irgend einem Athoskloster, wie ich Byz. Z. IV 577 vermutete, sondern - im Pantokratorkloster in Konstantinopel. Der Zeitpunkt freilich, wann or hier eintrat und den Namen Gennadios annahm, ist nicht mit voller Sicherheit zu bestimmen. Aber Tryphon E. Euangelides ${ }^{2}$ ) wird Recht haben, wenn er ihn zwischen das Ende des Jahres 1449 und den Anfang des Jahres 1451 setzt. Er schliefst das einmal aus einer an den nach dem Tode seines kaiserlichen Bruders Johannes Paläologos (31. Oktober 1449) noch im Peloponnes weilenden Despoten Konstan-

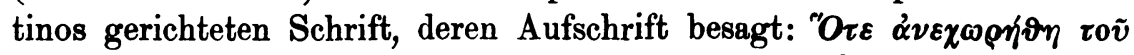

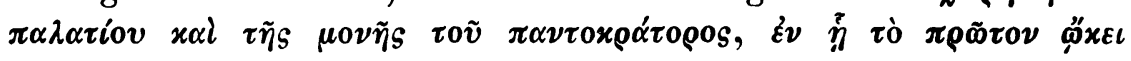

1) Gibbon, History of the decline and fall. of the Roman empire VIII 68, S. 282 nach Sporschils Ubersetzung.

2) In seiner aus Athen mir freundlichst zugesandten Schrift $\Gamma \varepsilon v v \alpha^{\prime} \delta\left\llcorner\right.$ s $\beta^{\prime} \delta$

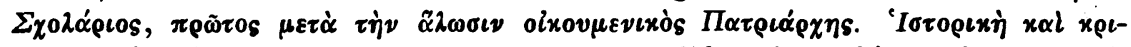

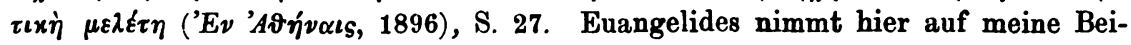
träge „Zu Georgios Scholarios" (Byz. Zeitschr. IV 561-580) und „Zu Marcus Eugenicus von Ephesus" (Zeitschr. f. Kirchengesch. XII 91-116) wiederholt Bezug. Gerade die letztere, aus dem Jahre 1887 stammende Arbeit ist, wie ich nachträglich zu meiner Befriedigung aus der Anm. 1 zu S. 7 ersehe, von Meletios

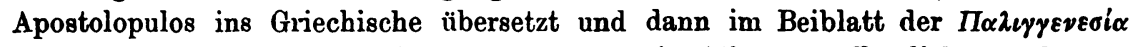
1895 in zehn aufeinander folgenden Nummern in Athen veröffentlicht worden: 


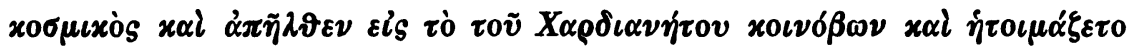

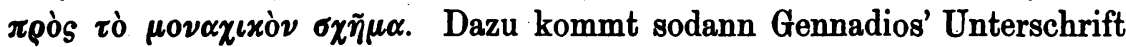
unter dem von der Kirche der Hauptstadt am 18. Januar 1451 an die

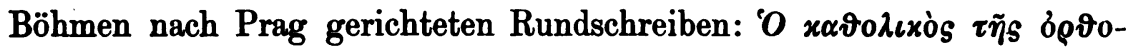

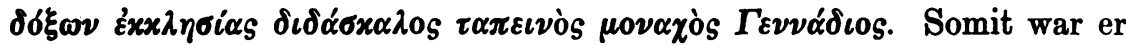
damals bereits als Mönch im Pantokratorkloster. Eine Reihe von Kundgebungen des vielgeplagten Mannes ist nun gerade von hier ausgegangen. Nur die letzte derselben, den von Gennadios an die Bürger von Byzanz gerichteten Brief vom 27. November (nicht 26. November, wie a. a. O. S. 576 steht) 1452 , habe ich Byz. Z. IV 576-577 nach Demetrakopulos zum ersten Male mitgeteilt und seinen Inhalt für die Lebensgeschichte des Georgios Scholarios, besonders für den Nachweis der Einheit der Persönlichkeit des anfänglich der Kirchenvereinigung freundlich, dann aber entschieden feindlich gesinnten Wortführers der Hellenen nutzbar zu machen gesucht. Es ist wichtig, auch die anderen in ihrer zeitlichen Folge kennen zu lernen. Von niemandem - soviel ich sehe - bisher beachtet, finden sie sich in Demetrakopulos' "Geschichte der Kirchenspaltung", der sie, mit einer Ausnahme, zuerst aus Handschriften veröffentlicht hat.

Das erste Wort, das Gennadios von seiner Zelle aus an seine Mitbürger richtete, nimmt unmittelbar Bezug auf das Erscheinen des Kardinals Isidoros. Es steht im Cod. Mosqu. 208 S. 549 (Dem. S. 163),

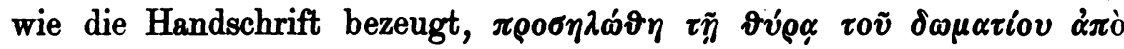

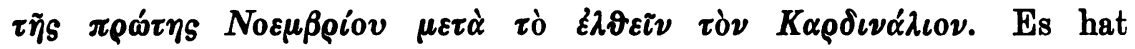
folgenden Wortlaut:

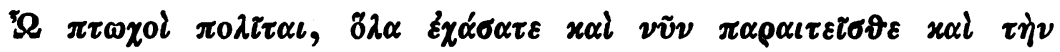

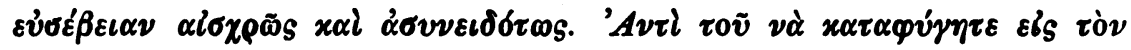

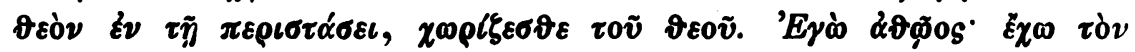

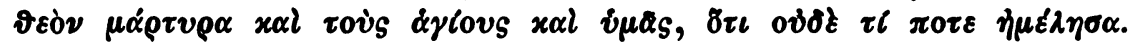

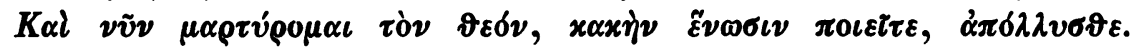

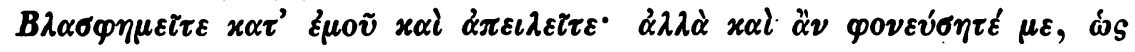

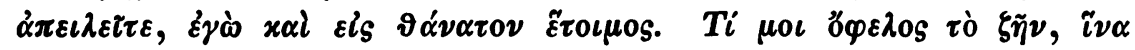

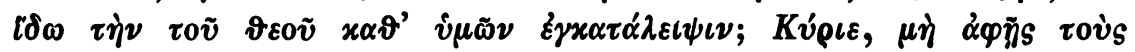

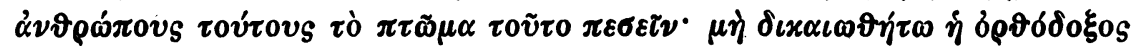

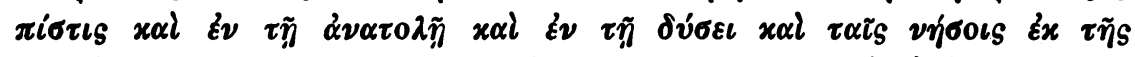

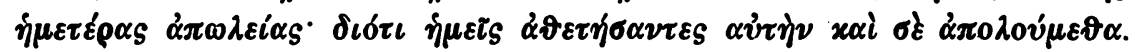

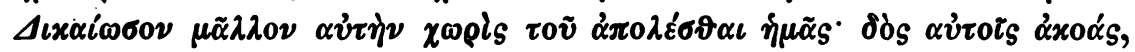

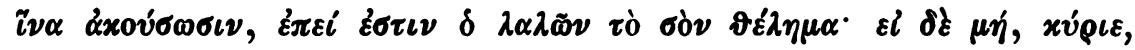

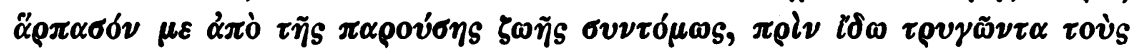

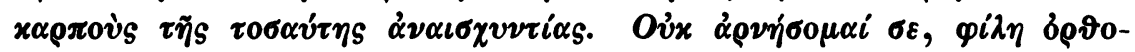

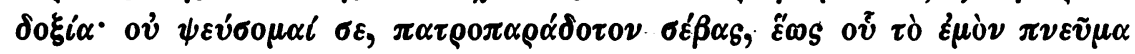




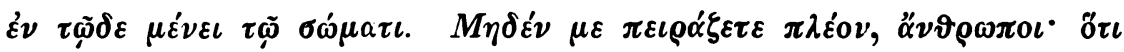

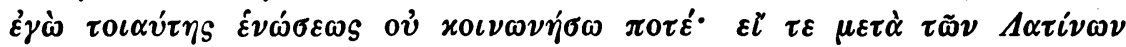

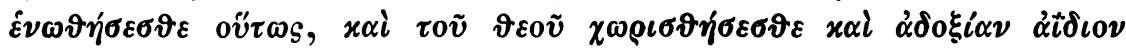
$\dot{\boldsymbol{v} \pi} \boldsymbol{\pi} \sigma \tau \dot{\eta} \sigma \varepsilon \sigma \vartheta \varepsilon$.

Die Worte erinnern an die Gedanken, die Georgios Scholarios schon in seiner an den Grofs-Dux gerichteten Schrift (Byz. Z. IV 573-575) niedergelegt hatte. Sie gewähren uns einen erschütternden Einblick in die durch die Gefahr der Lage, in der sogar sein eigenes Leben bedroht ist, und durch das Gefühl der Ohnmacht und der Vergeblichkeit des eigenen Strebens und Ringens gegen die Römerfreunde verdüsterte Stimmung des treuen Verteidigers der griechischen Lehre.

Gennadios schien vergeblich zu warnen. Die Verhandlungen nahmen ihren Fortgang. Nach wenigen Tagen schon wurden die Geistlichen der Hauptstadt in den Palast Xylalas berufen. Wer sollte ihr Sprecher sein? Nur Gennadios schien dazu imstande zu sein. Man bat ihn, sein Kloster zu verlassen und in den Palast zu kommen. Er aber lehnte dies ab, sandte vielmehr am 15. November durch Vermittelung Silvester Syropulos', des Geschichtschreibers des Florentiner Konzils, und Theodoros Agallianos' ein ausführliches Schreiben an die Versammlung, das Demetrakopulos in Abschrift aus einer noch nicht herausgegebenen Handschrift vom russischen Archimandriten Amphilochios erhielt und um seiner sprachlich merkwürdigen, $d$. h. vielfach vulgärgriechischen Form und seines geschichtlich höchst wertvollen Inhalts willen in seiner "Geschichte der Kirchenspaltung" S. 166-172 zum ersten Male veröffentlicht hat. Ich verweise auf diese Stelle; der Brief ist zu umfangreich, um hier vollständig abgedruckt zu werden. Nur einige Gedanken mögen hervorgehoben werden, sofern sie sich mit schon früher mitgeteilten berühren oder sie ergänzen. Ich setze zunächst den Anfang her, weil er die Gründe für Gennadios' Zurülkhaltung enthält:

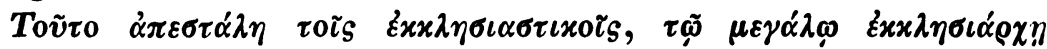

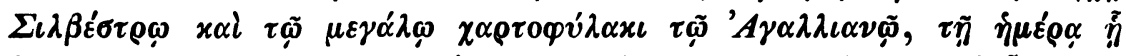

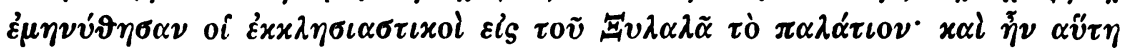

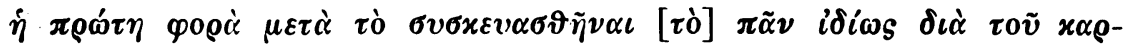

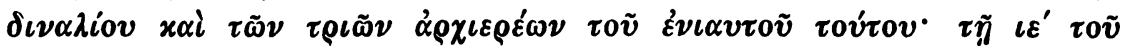

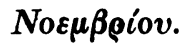

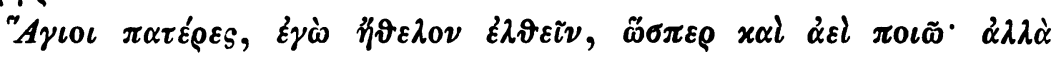

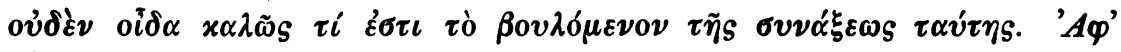

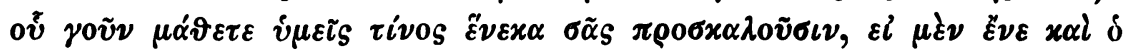

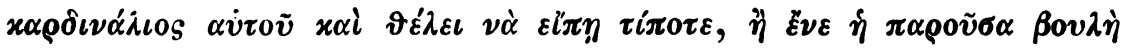

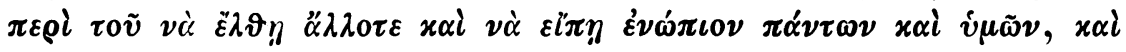




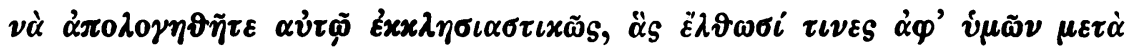

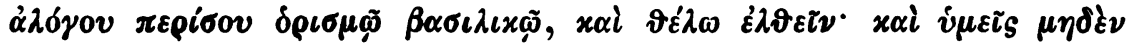

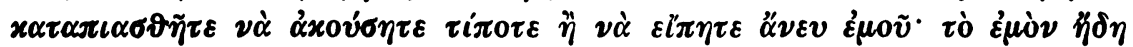

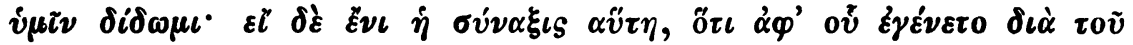

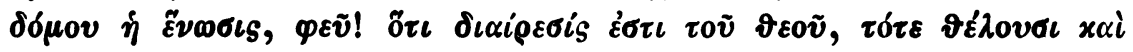

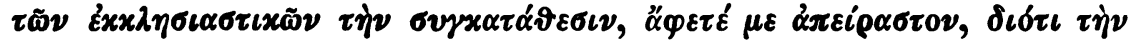

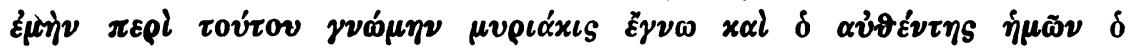

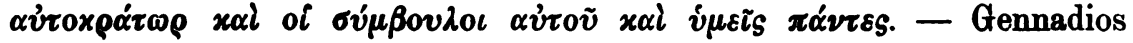
kann sich mit Recht, wie er das ja schon in der von mir Byz. Z. IV 573-575 inhaltlich erläuterten Schrift gethan, auf die Vergangenheit berufen. Die Lage ist ja Rom gegenüber immer dieselbe geblieben.

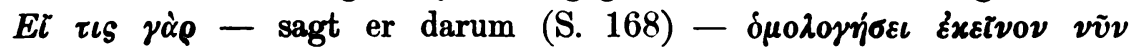

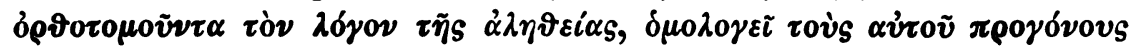

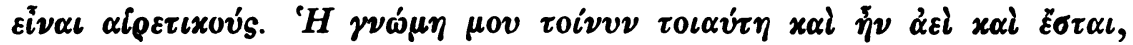

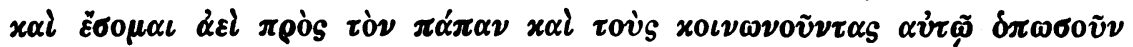

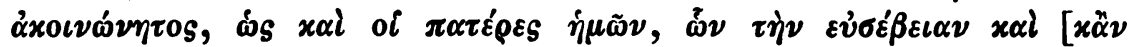

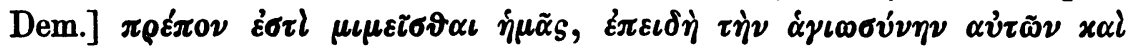

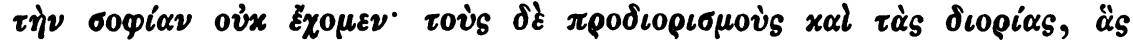

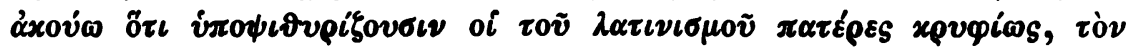

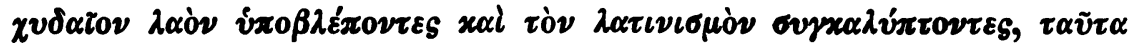

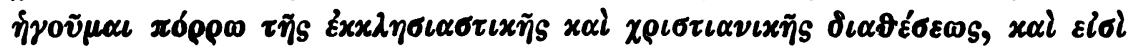

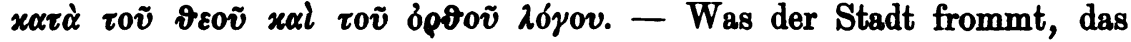

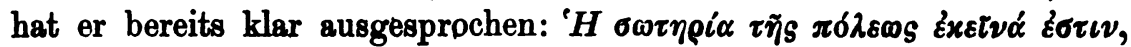

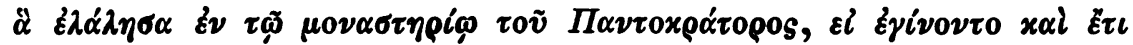

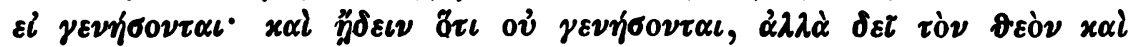

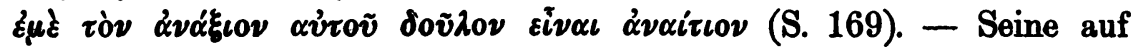
den Grofs-Dux ${ }^{1}$ ) gesetzten Erwartungen (vgl. Byz. Z. IV 575) sind ihm

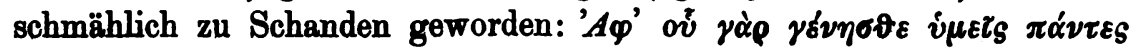

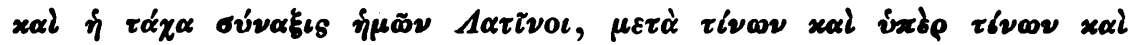

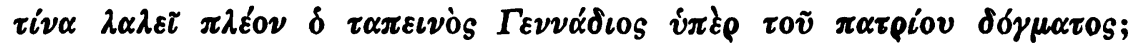

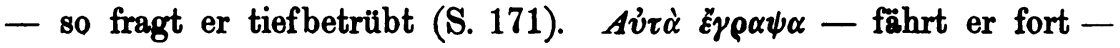

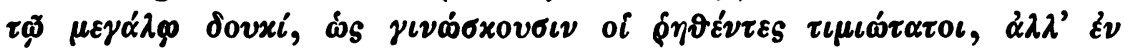

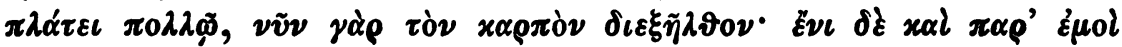

1) Es ist an den wackeren Lukas Notaras zu denken, nicht etwa an Demetrios, des Kaisers Bruder (Byz. Z. IV 575), der damals Despot im Peloponnes war. Notaras ist jener unglückliche Grofswürdenträger, der, bei der Eroberung der Stadt zuerst von Mohammed II begnadigt und gütig behandelt, dann, als er sich weigerte, seinen schőnen vierzehnjährigen Sohn dem Sultan zum Gastmahl zu schicken, auf Befehl des unmenschlichen Siegers samt seinem ältesten Sohne und Schwiegersohne enthauptet wurde. Vgl. Gibbon a. a. O. VIII 68 S. 316 und Hertzberg a. a. O. S. $\mathbf{5 9 0}$. 


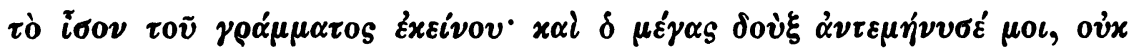

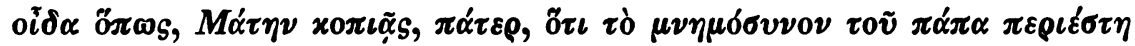

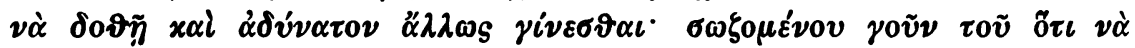

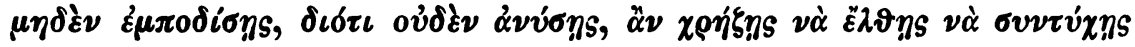

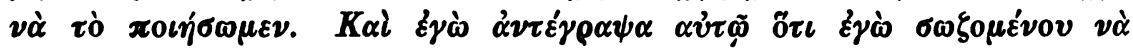

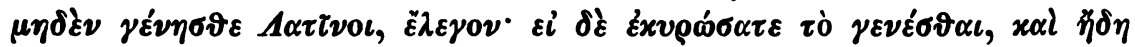

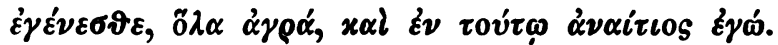

Ist es nicht ergreifend zu hören und zu sehen, wie vergeblich Gennadios sich abgemüht hat? Die gleichen Gedanken, die gleiche Stimmung wehen uns endlich aus dem zuvor schon (Byz. Z. IV 576-577) behandelten Schreiben vom 27. November an die Bürger von Byzanz entgegen. Freilich, die Kircheneinigung, die Kardinal Isidoros, durch die immer furchtbarer sich nähernde Türkengefahr in seinem Vorgehen begünstigt, nachdrücklich betrieb, kam nicht zustande. Schon hatte er, wie es schien, den Kaiser für sich, da dieser mit den gefügigsten Geistlichen und Laien die Vereinigungsurkunde, wie sie auf dem Konzil zu Florenz genehmigt worden war, unterzeichnete, allerdings mit dem Vorbehalt, dafs sie nach Aufhören der Kriegsgefahr von Seiten der Türken einer nochmaligen Durchsicht unterzogen werden sollte. Ja es kam am 12. Dezember in der Sophienkirche zur Abendmahls- und Gebetsgemeinschaft in einem feierlichen, von Lateinern und Hellenen gemeinsam abgehaltenen Gottesdienst, bei welchem die Namen des Papstes Nikolaus V und des in Rom weilenden Patriarchen Gregorios neben einander genannt wurden. Aber während dieser Vorgänge in der Sophienkirche erschienen die Gegner der Kircheneinigung bei Gennadios Scholarios im Pantokrator-Kloster mit der Frage: Was sollen wir thun? Wiederum verliefs Gennadios seine Zelle nur, um seine Meinung, seine letzte Warnung schriftlich an die Thür zu heften. Sie lautete nach Johannes Dukas (S. 254 der Bonn. Ausg.):

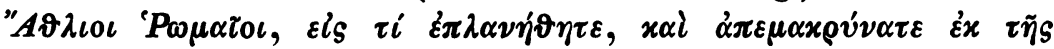

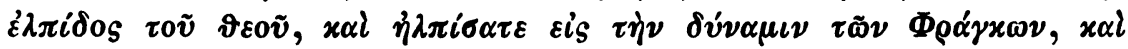

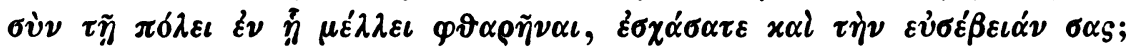

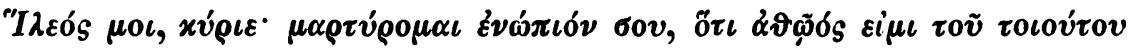

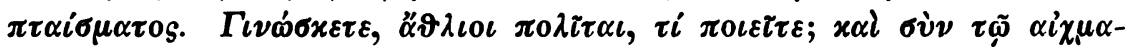

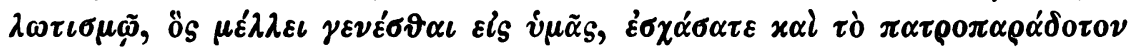

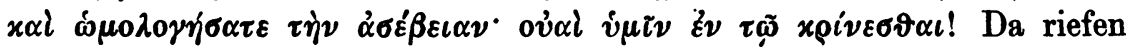
Geistliche und Laien das Verdammungsurteil über das Florentiner Konzil und dessen Anhänger, und das aus dem Hof des PantokratorKlosters in dichten Scharen strömende Volk schrie den Fluch aus über diejenigen, welche der Einigung zugestinnt hatten. Der Kircheneinigungsversuch vom Jahre 1439 war damit endgültig gescheitert. 
586 I. Abteilung. J. Drh̆seke: Zum Kircheneinigungsversuch des Jahres 1439

Die Ereignisse drängten sich jetzt. Das neue Jahre brachte die letzte, blutige Entscheidung. Konstantinopel ward am 29. Mai 1453 von den Türken erobert, Kaiser Konstantinos, der alle Pflichten des Feldherrn und des Kriegers bis zum letzten Atemzuge getreulich erfüllte, fiel als der letzte Paläologe mit allen Edlen seiner nächsten Umgebung heldenmütig kämpfend von unbekannter Hand, seine Leiche wurde von einem Berge von Erschlagenen begraben.

Wandsbeck.

Johannes Drăseke. 